\title{
A New Approach to Assess the Physical Demands of Young Tennis Players: Inertial Movement Analysis. Preliminary Data
}

\author{
Carlos Galé-Ansodi*, Julen Castellano, Oidui Usabiaga \\ Department of Physical Education and Sport, Faculty of Physical Activity and Sport Sciences, University of the Basque Country, Vitoria-Gasteiz, Spain
} Submission: September 19, 2019; Published: October 15, 2019

"Corresponding author: Carlos Galé-Ansodi, Department of Physical Education and Sport, Faculty of Physical Activity and Sport Sciences, University of the Basque Country, Vitoria-Gasteiz, Spain

\section{Abstract}

In recent years, there has been an increased interest around the use of wearable microsensor technology in order to improve the knowledge about activities patterns such as accelerations, decelerations, jumps and changes of direction. This complex information is a result of accelerometers, magnetometers and gyroscopes and is processed with an advanced algorithm to provide Inertial Movement Analysis (IMA). This technology may also calculate the magnitude and the direction of an agility action, classifying events within intensity and direction. Therefore, the aim of this study was to describe the physical profile of elite young tennis players focusing in the "IMA" patterns. Twenty female young high-level tennis players took part in the study (14.3 age \pm 0.8 years). All tennis players were tracked at least in two matches-play ( $\mathrm{n}=62)$ on hard court. Twelve Portable GPS devices (Minimax X S4, Catapult Innovations) operating at a sampling frequency of $10 \mathrm{~Hz}$ were used to collect velocity data. The GPS unit contains a tri-axial piezoelectric linear accelerometer (Kionix: KXP94) sampling at a frequency of $100 \mathrm{~Hz}$. The most common axis of movement was the vertical (V_axis:39.3 $\pm 1.9 \%$ ). On the other hand, lateral displacement were the most common movements of tennis players (right:6.4 \pm 1.2 effort $\cdot$ min 1; left: $6.3 \pm 1$. effort· min 1). Furthermore, low accelerations were the most common acceleration pattern in tennis (low accelerations: $7.4 \pm 2.3$ effort $\cdot \mathrm{min}-1)$, whereas, the high intensity accelerations $(>1.0 \mathrm{~m} \cdot \mathrm{s}-2)$ were the less frequent (high accelerations: $5.1 \pm 1.6$ effort-min-1). Therefore, further literature should focus on "IMA" patterns to improve tennis players' performance. .

Keywords: IMA; Racquet Sports; Acceleration; Direction Movement; GPS

\section{Introduction}

In recent years, scientists have used Global Position System (GPS) devices to investigate the physical demands in different sports Baiget [1], Cummins, Orr, O Connor, West [2]. Nowadays, there has been an increased interest around the use of wearable microsensor technology in order to improve the knowledge about activities patterns such as accelerations, decelerations, jumps (e.g., steps in tennis) and changes of direction Akenhead, Hayes, Thompson, French [3], Dellaserra, Gao, Ransdell [4]. This complex information is a result of accelerometers, magnetometers and gyroscopes and is processed with an advanced algorithm to provide Inertial Movement Analysis (IMA) - a simple way of expressing the most complex athlete movements Catapult-Sports [5]. This approach combines accelerometer with gyroscope and magnetometer data, allowing the examination of accelerations with respect to movement directions. In this line, accelerometers are motion sensors that can detect linear acceleration along one or several axis, whereas gyroscopes are motion sensors that can detect angular velocity about one or several axis Yang, Hsu [6]. Finally, a magnetometer is a sensor that detects the earth's magnetic field; specifically, "it can be used to correct the orientation to the gyroscope" Aminian, Najafi [7].

This technology is termed like Inertial movement analysis "IMA" and it may also calculate the magnitude and the direction of an agility action, classifying events within intensity, and distinguish between forward (-45 to 45), backward (-135 to 135$)$, left lateral ( -135 to -45 ) and right lateral (45 to 135 ) events Catapult-Sports [5] (Figure 1). In this way, researchers and coaches can now evaluate the activity profiles of players in different team sport matches Gaudino, Alberti, Iaia [8], Holme [9]. However, there are no published studies to date in tennis matchplay that have used "IMA" to analyses the physical demands of tennis-players. For this reason, the authors consider appropriate to continue exploring the physical demands of elite young tennis players in match-play focusing in "IMA" patterns. Therefore, the aim of this study was to describe the physical profile of elite young tennis players paying attention in the "IMA" patterns. 


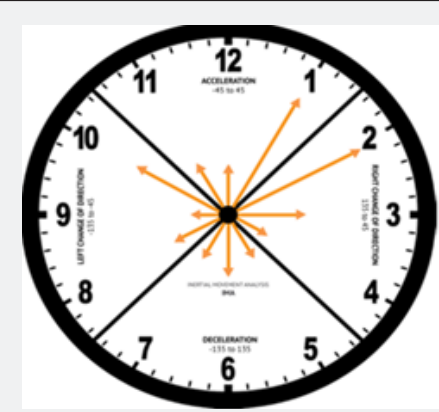

Figure 1: Illustrates calculation of IMA direction and how events are appearing on the radar plots.

\section{Methods}

Twenty female young high-level tennis players took part in the study (14.3 age \pm 0.8 years). All tennis players were tracked at least in two matches-play $(n=62)$, carried out on hard court from 16:00 to 20:00 hours in Aragon Tennis Federation facilities in December 2016. The weather conditions during matches-play were similar $\left(5{ }^{\circ} \mathrm{C}-9{ }^{\circ} \mathrm{C}\right.$ and $54 \%-62 \%$ relative humidity). Twelve Portable GPS devices (Minimax X S4, Catapult Innovations) operating at a sampling frequency of $10 \mathrm{~Hz}$ were used to collect velocity data. The GPS unit contains a tri-axial piezoelectric linear accelerometer (Kionix: KXP94) sampling at a frequency of 100 Hz. Data were collected during what were considered to be good GPS conditions in terms of the weather and satellite conditions. The players wore a special harness underneath the playing clothes between both scapulas. The reliability and accuracy of the devices used in this study in short distance exercises has been assessed in previous works Castellano, Casamichana, Calleja-González, San Román, Ostojic [10].

In this way, the validity and reliability of inertial movement analysis during sprinting and changes of directions were positively evaluated Meylan, McKean [11]. Moreover, GaléAnsodi, Langarika-Rocafort, Usabiaga, Castellano [12] have shown the reliability of this type of GPS was adequate also in tennis tasks. The tennis players and their parents were informed of the purpose, procedures, and potential risks involved in this study, and written informed consent was provided by both, due to players were under the age of 18 . The Ethic Committee of the University of the Basque Country (UPV/EHU) gave its institutional approval of the study. All the statistical analysis was performed using SPSS 20.0 for Windows. The data are expressed as mean, percentage $(\%)$ and standard deviation $( \pm$ SD).

\section{Results}

As can be seen in Figure 2 the players' acceleration patterns were not distributed equally in the three axis of movement. The most common axis of movement was the vertical (V_axis:39.3 $\pm 1.9 \%$ ), overcoming the movements developed by tennis players from side to side on the tennis court (L_axis: $31.8 \pm 1.7 \%$ ). Finally, the perpendicular movements to the baseline had less predominance than the previous results (AP_axis: $28.9 \pm 1.3 \%$ ) (Figure 2). On the other hand, the number of accelerations per minute taking into the direction of movement were analysed too (Figure 3). Lateral displacement parallel to back line were the most common movements of tennis players (right:6.4 \pm 1.2 effort $\cdot \min 1$; left:6.3 \pm 1 . effort $\cdot \min 1$ ). However, tennis players developed a smaller number of efforts to forward $(4.7 \pm 0.7$ effort·min-1) and to backward (1.8 \pm 0.3 effort $\cdot$ min- 1$)$.

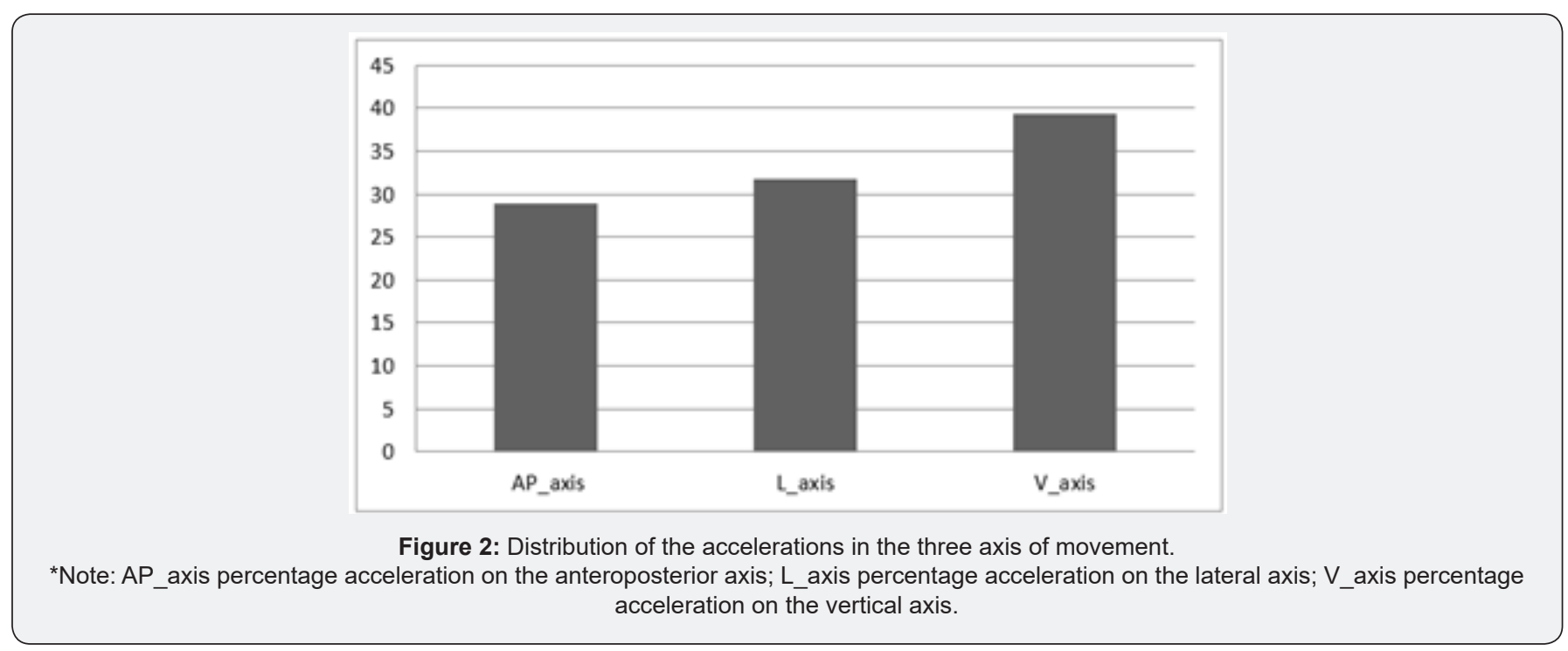


Regarding the acceleration's intensity, the Figure 3, shows that low accelerations performed between $0.0-0.5 \mathrm{~m} \cdot \mathrm{s}-2$ were the most common acceleration pattern in tennis (low accelerations: $7.4 \pm 2.3$ effort $\cdot \mathrm{min}-1)$, whereas, the high intensity accelerations $(>1.0 \mathrm{~m} \cdot \mathrm{s}-2)$ were the less frequent (high accelerations: $5.1 \pm 1.6$ effort-min-1). However, attending to the direction of movement, the accelerations from side to side (right:6.4 \pm 2.0 effort $\cdot$ min- 1 and left: $6.3 \pm 2.1$ effort $\cdot \min -1)$ were higher than the rest of accelerations at low $(0.0-0.5 \mathrm{~m} \cdot \mathrm{s}-2)$ and at medium $(0.5-1.0 \mathrm{~m} \cdot \mathrm{s}-$ 2) intensity. However, taking into account only the high intensity accelerations, the forward accelerations were higher than lateral and back accelerations (forward: $2.5 \pm 1.1$; right:1.13 \pm 0.3 ; left:1.0 \pm 0.3 ; back: $0.4 \pm 0.2$ all in effort $\cdot \min 1$ ) (Figure 3).

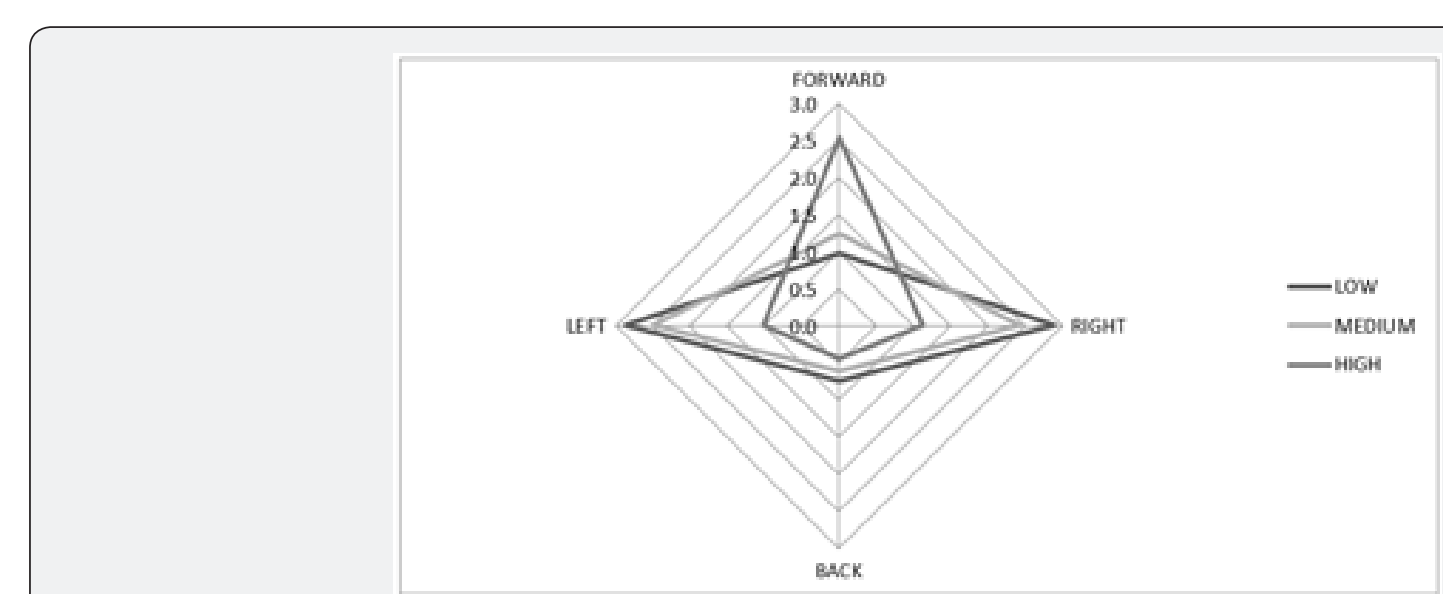

Figure 3: Number of accelerations per minute at different intensity ranges in all directions of movement.

*Note: Low: accelerations performed between $0.0-0.5 \mathrm{~m} \bullet \mathrm{s}^{-2}$; Medium: accelerations performed between $0.5-1.0 \mathrm{~m} \cdot \mathrm{s}^{-2} ;$ High: accelerations performed at $>1.0 \mathrm{~m} \cdot \mathrm{s}^{-2}$.

\section{Discussion}

To the best of our knowledge, this is the first study describing the "IMA" patterns of young tennis players in official matches using GPS. The results showed that the most common plane of movement in tennis players was the vertical axis. This fact could be due to tennis players use to take off their feet from the ground to hit the ball and bending to start running, according to Davies, Young, Farrow and Bahnert [13], who showed that football players performed greater values on the vertical axis of the movement as a result of the number of sprints done. Therefore, it seems to be accepted that the accelerations and sprints collaborate to achieve high values in the displacements realized on the vertical axis. Taking into account the number of accelerations, the results showed that lateral displacement overcome the rest of movements developed in other directions.

These results were similar to Weber, Pieper y Exler [14], who founding that the $71,8 \%$ of tennis players' movements were covered parallel to back line. In this line, Fernandez-Fernandez, and Méndez-Villanueva [15] concluded that tennis players developed four changes of direction per each point. Finally, there were a great number of high accelerations (>1.0 m-s-2) which took place to forward. On the contary, lateral accelerations were more common than forward acceleration at low and medium intensity, according to Hoppe, Baumgart, and Freiwald [16] where adults tennis players covered a great number of accelerations $(>4 \mathrm{~m} \cdot \mathrm{s}-2)$ to forward. Further literature should focus on "IMA" patterns to improve tennis players' performance because a wide knowledge about this kind of patterns of movement would allow coaches to design specific training programs.

\section{References}

1. Baiget-Vidal E (2011) Metodología del entrenamiento de la resistencia específica en el tenis de competición. Revisión y propuesta. Training methods for specific endurance in competitive tennis. Review and proposal. Cultura, Ciencia y Deporte 6(16): 45-54.

2. Cummins C, Orr R, O'Connor H, West C (2013) Global positioning systems (GPS) and microtechnology sensors in team sports: A systematic review. Sports Med 43(10): 1025-1042.

3. Akenhead R, Hayes P, Thompson K, French D (2013) Diminutions of acceleration and deceleration output during professional football matchplay. J Sci Med Sport 16(6): 556-561.

4. Dellaserra CL, Gao Y, Ransdell L (2014) Use of integrated technology in team sports: a review of opportunities, challenges, and future directions for athletes. J Strength Cond Res 28(2): 556-573.

5. Catapult-Sports (2013) Sprint Help - Inertial Movement Analysis (IMA): For Sprint 5.8 and subsequent releases.

6. Yang CC, Hsu YL (2010) A review of accelerometry-based wearable motion detectors for physical activity monitoring. Sensors (Basel) 10(8): 7772-7788.

7. Aminian K, Najafi B (2004) Capturing human motion using body-fixed sensors: outdoor measurement and clinical applications. Computer Animation and Virtual Worlds 15(2): 79-94.

8. Gaudino P, Alberti G, Iaia FM (2014) Estimated metabolic and mechanical demands during different small-sided games in elite soccer players. Human Movement Science 36: 123-133.

9. Holme BR (2015) Wearable microsensor technology to measure physical activity demands in handball. A reliability study of Inertial 
movement analysis and Player Load. Master Thesis in Sport Sciences. Department of Physical Performance Norwegian School of Sport Sciences. Oslo, United Nations.

10. Castellano J, Casamichana D, Calleja -González J, San Román J, Ostojic S (2011) Reliability and accuracy of $10 \mathrm{~Hz}$ GPS devices for short-distance exercise. Journal of Sports Science and Medicine 63(1): 233-234.

11. Meylan, McKean (2013) The validity and reliability of inertial movement analysis during sprinitng and change of direction in elite women's soccer players. Calgary, Conference. Canadian Sport Institute - Pacific, Canada.

12. Galé-Ansodi C, Langarika-Rocafort A, Usabiaga O, Castellano J (2016) New variables and new agreements between $10 \mathrm{~Hz}$ global positioning system devices in tennis drills. Journal of Sports Engineering and Technology p. 1-3.
13. Davies MJ, Young W, Farrow D, Bahnert A (2013) Comparison of agility demands of small-sided games in elite Australian football. Int J Sports Physiol Perform 8(2): 139-147.

14. Weber K, Pieper S, Exler T (2007) Characteristics and significance of running speed at the Australian Open 2006 for training and injury prevention. Medicine and Science in Tennis 12: 14-17.

15. Fernández-Fernández J, Sanz-Rivas D, Méndez-Villanueva A (2009) A review of the activity profile and physiological demands of tennis match play. Journal Strength Conditioning Research 31(4): 15-26.

16. Hoppe MW, Baumgart C, Freiwald J (2015) Do running activities of adolescent and adult tennis players differ during play? International Journal of Sports Physiology and Performance 11(6): 793-801.

\section{Your next submission with Juniper Publishers will reach you the below assets}

- Quality Editorial service

- Swift Peer Review

- Reprints availability

- E-prints Service

- Manuscript Podcast for convenient understanding

- Global attainment for your research

- Manuscript accessibility in different formats

( Pdf, E-pub, Full Text, Audio)

- Unceasing customer service

Track the below URL for one-step submission https://juniperpublishers.com/online-submission.php 